Accepted manuscript (c) <2020>. This manuscript version is made available under the CC-BY-NC-ND 4.0 license http://creativecommons.org/licenses/by-nc-nd/4.0/

Finally published in Nanoscale 2018, 10, 2820

\title{
Free-electron-laser coherent diffraction images of individual drug-carrying liposome particles in solution
}

Chi-Feng Huang, ${ }^{a}$ Keng S. Liang, ${ }^{a}$ Tsui-Ling Hsu, ${ }^{b}$ Tsung-Tse Lee, ${ }^{a}$ Yi-Yun Chen, ${ }^{a}$ ShunMin Yang, ${ }^{a}$ Hsiang-Hsin Chen, ${ }^{a}$ Shih-Hsin Huang, ${ }^{c}$ Wei-Hau Chang, ${ }^{c}$ Ting-Kuo Lee, ${ }^{a}$ Peilin Chen, ${ }^{d}$ Kuei-En Peng, ${ }^{e}$ Chien-Chun Chen, ${ }^{e}$ Cheng-Zhi Shi, ${ }^{f}$ Yu-Fang Hu, ${ }^{f}$ Giorgio Margaritondo, ${ }^{\mathrm{g}}$ Tetsuya Ishikawa, ${ }^{\mathrm{h}}$ Chi-Huey Wong, ${ }^{\mathrm{b}}$ and $\mathrm{Y}$. $\mathrm{Hwu}^{\mathrm{a}}$

\footnotetext{
a Institute of Physics, Academia Sinica, Taipei 115, Taiwan.Phhwu@sinica.edu.tw

${ }^{b}$ Genomics Research Center, Academia Sinica, Taipei 115, Taiwan

c Institute of Chemistry, Academia Sinica, Taipei 115, Taiwan

${ }^{d}$ Research Center for Applied Sciences, Academia Sinica, Taipei 115, Taiwan

e Department of Physics, National Sun Yat-sen University, Kaohsiung 804, Taiwan

${ }^{f}$ TTY Biopharm Co., Ltd, Taipei 115, Taiwan

9 Ecole Polytechnique Fédérale de Lausanne, Lausanne, CH-1025, Switzerland

h Japan Synchrotron Radiation Research Institute/Spring-8, Hyogo 679-5198, Japan
}

\begin{abstract}
Using the excellent performances of a SACLA (RIKEN/HARIMA, Japan) X-ray free electron laser (X-FEL), coherent diffraction imaging (CDI) was used to detect individual liposome particles in water, with or without inserted doxorubicin nanorods. This was possible because of the electron density differences between the carrier, the liposome, and the drug. The result is important since liposome nanocarriers at present dominate drug delivery systems. In spite of the low cross-section of the original ingredients, the diffracted intensity of drugfree liposomes was sufficient for spatial reconstruction yielding quantitative structural information. For particles containing doxorubicin, the structural parameters of the nanorods could be extracted from CDI. Furthermore, the measurement of the electron density of the solution enclosed in each liposome provides direct evidence of the incorporation of ammonium sulphate into the nanorods. Overall, ours is an important test for extending the $\mathrm{X}-\mathrm{FEL}$ analysis of individual nanoparticles to low cross-sectional systems in solution, and also for its potential use to optimize the manufacturing of drug nanocarriers.
\end{abstract}

\section{Introduction}

The first X-FELs ${ }^{1-3}$ are revolutionizing structural investigations at the submicron level, ${ }^{4-7}$ yielding detailed information on individual particles. This was notably demonstrated for metal nanoparticles and viruses of a relatively large size ${ }^{7,8}$ Here we show that the rapid progress of X-FELs and detectors now enables the imaging of individual liposome particles of $\sim 100$ $\mathrm{nm}$ size and in water, ${ }^{9}$ even though they consist in principle of weakly scattering biomolecules, and of their carried drugs..$^{10-12}$ The diffracted intensity was specifically sufficient for $\mathrm{CDI}$ reconstruction ${ }^{13-16}$ yielding quantitative information on individual liposomes. 
Liposomes are vesicles composed of lipids, used to deliver nutrients or drugs. ${ }^{17-19}$ Their excellent, size-dependent delivery properties are specifically employed for carrying doxorubicin, a widely used anticancer drug. ${ }^{17,20-24}$ Further improvements, specifically those concerning their size and homogeneity, ${ }^{25,26}$ would be highly desirable for medical and other health-related applications, not to mention compliance with the drug-certification requirements. However, these improvements necessitate a detailed knowledge of the structural properties of liposomes that is hard to achieve. It is indeed difficult to identify the shape, size and drug uptake of liposomes by standard imaging methods, particularly in a natural liquid environment.

We solved these problems by using the CDI of individual nanoparticles in solution, based on X-FEL pulses. The experiments were performed with a specially designed sample holder to analyze liposomes in a liquid environment - an essential condition for a realistic impact on the optimization of drug delivery.

Our quantitative X-FEL CDI results were generally consistent with cryo-electron microscopy (cryo-EM) data ${ }^{27-30}$ and with the small-angle X-ray scattering (SAXS) analysis of the diffraction patterns. However, our approach offers marked advantages.

Indeed, cryo-EM cannot be implemented in the natural liquid environment. Conventional SAXS on liquid solutions cannot be applied to anisotropic nanorods with non-uniform size distribution, as in the present case. These are limitations of general significance, since the typical structure of drug-carrying nanoparticles is indeed anisotropic and not uniform. Similar limitations also affect other imaging techniques: for example, dynamic light scattering ${ }^{31}$ provides useful information, but performs averages over many particles and cannot detect the drug rods inside them.

\section{Results and discussion}

Fig. 1 shows the cryo-EM images of blank and doxorubicin-containing liposomes. Note that the therapeutical use is only effective and officially approved for rod-shaped drug particles inside liposomes within a narrow size range. Fig. $1 \mathrm{~b}$ and $\mathrm{c}$ reveal significant deviations from this standard: the liposome size ranges from $\sim 80 \mathrm{~nm}$ to $>250 \mathrm{~nm}$ (and requires size filtering in the industrial drug production process). Furthermore, whereas some liposomes contain doxorubicin with the correct shape (i.e., rods - see for example the arrow-marked particle in Fig. 1b), others contain doxorubicin with unacceptable shapes. Furthermore, some liposomes contain no doxorubicin at all, and others are enclosed in larger liposomes. These characteristics cannot be detected by particle-averaging techniques such as SAXS whereas they are crucial for therapy, its optimization and the mandatory controls of industrial manufacturing for human use.

Fig. 2 shows representative CDI results of two individual liposomes with no doxorubicin; Fig. 2(a) is a diffraction pattern, Fig. $2 b$ the pattern after processing with the Guided Hybrid InputOutput $(\mathrm{GHIO})^{15}$ method and Fig. 2(c) is the resulting reconstruction. In this widely used image-processing technique, a circular constraint improves the appearance of the images but it does not in any way alter the essential geometric features. Fig. 2(d) and (e) show the diffraction pattern and the reconstruction of another individual liposome. From the reconstructions, we directly extracted the size of each liposome, $\sim 100 \pm 20 \mathrm{~nm}$ in both cases. 

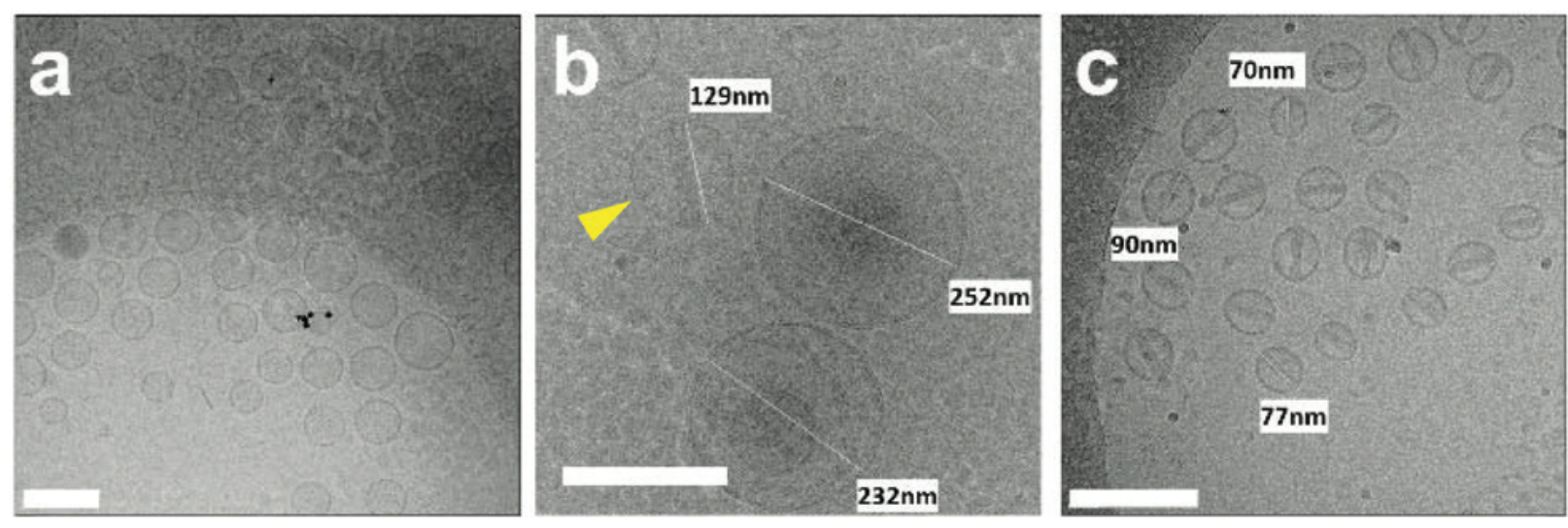

Fig. 1 Cryo-electron microscopy images of liposome particles. (a) Blank and (b) doxorubicin-containing liposomes (the black dots in (a) are Au nanoparticles decorating one of the liposomes). (c) Processed doxorubicin-containing liposome compliant with the human therapy require- ments, with a more uniform size and shape. Scale bars $=200 \mathrm{~nm}$.
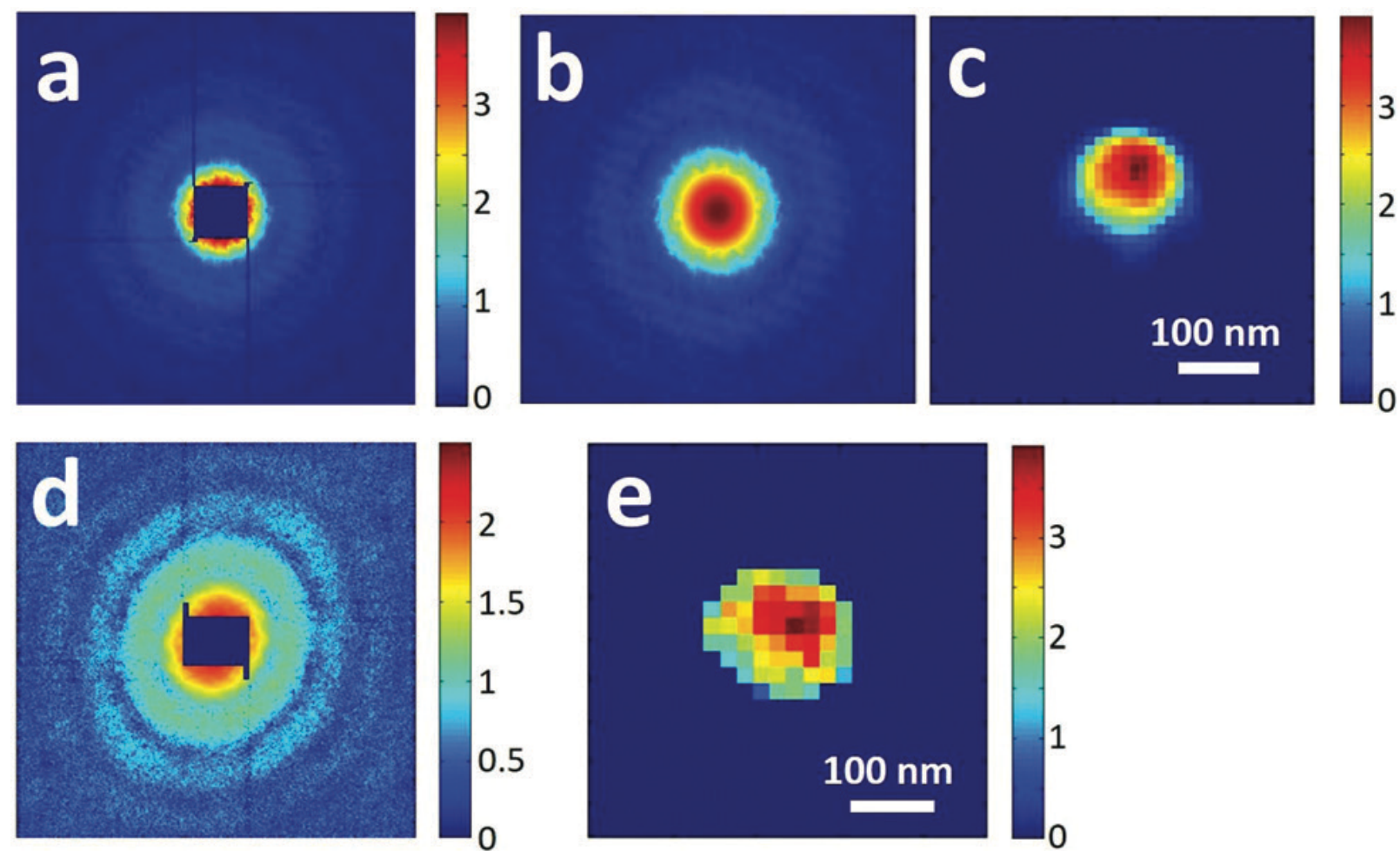

Fig. 2 Representative CDI patterns for individual liposomes with no doxorubicin. (a)-(c) show typical results of one liposome: (a) diffraction pattern; (b) the same pattern after GHIO processing (initial reconstruc- tion); (c) the corresponding reconstructed image in real space (the error factor is 0.1741 ). Scale bars $=100 \mathrm{~nm}$. (d) and (e) Diffraction pattern and reconstructed image of another drug-free liposome. The color scales are in arbitrary units.

Concerning spatial resolution, for $\mathrm{CDI}$ it is limited by the largest detectable scattering vector, i.e., by the largest angle at which diffraction can be measured. Our present experimental setup can potentially achieve $10 \mathrm{~nm}$ resolution under optimized conditions. However, from small-angle-X-ray-scattering (SAXS-like) we estimate that the real resolution is $\sim 20 \mathrm{~nm}$. This, of course, is not the ultimate performance: with improved signal-to-noise levels better resolutions are feasible. 
Note that our time resolution (see below) was $10 \mathrm{fs}$, making factors like rotational or diffusion motions irrelevant. Other factors like the aggregation of nanoparticles are ruled out by the reconstructed images, in agreement with the results from cryo-EM.

To corroborate the CDI-derived particle sizes, we calculated SAXS-like curves from the diffraction patterns, by integrating over all directions the intensity for each $q$-value $(q=$ momentum transfer magnitude). Fig. $\mathrm{S} 1$ shows the curves so obtained for five different particles. The connection with real SAXS curves is only approximate, since our CDI patterns do not average over different particles. However, fits of our SAXS-like curves with a standard procedure ${ }^{32}$ reasonably corroborated the sizes extracted from CDI by giving results in the 100-200 nm range.

The results of Fig. 2 raise an intriguing question: how can CDI patterns be detected at all if the nominal scattering power of the constituents is weak? The answer can be provided by the practical composition of our liposomes. Indeed, they were produced by the same industrial process used for drug-containing liposomes. As a result, they are likely to include significant amounts of residual ammonium sulphate, $\left(\mathrm{NH}_{4}\right)_{2} \mathrm{SO}_{4}$, from the precursor solution. We estimated that the diffracted intensity of this compound in $2 \mathrm{M}$ solution, much lower than the saturation concentration ( 6 $\mathrm{M})$ which has an electron density $30-50 \%$ higher than water, can produce the contrast levels detected in our liposome patterns.

Fig. 3 shows the CDI results of four doxorubicin-containing liposomes in a liquid environment. The reconstructions clearly reveal rod-shaped doxorubicin particles. The lengths and widths seen in the reconstructions correspond, of course, to the twodimensional projections of the rods. The projection values are in the ranges of $40-80 \mathrm{~nm}$ (width) and $120-200 \mathrm{~nm}$ (length), and the aspect ratio is $0.25-0.6$. Such results are quite reasonable in the light of the cryo-EM data.
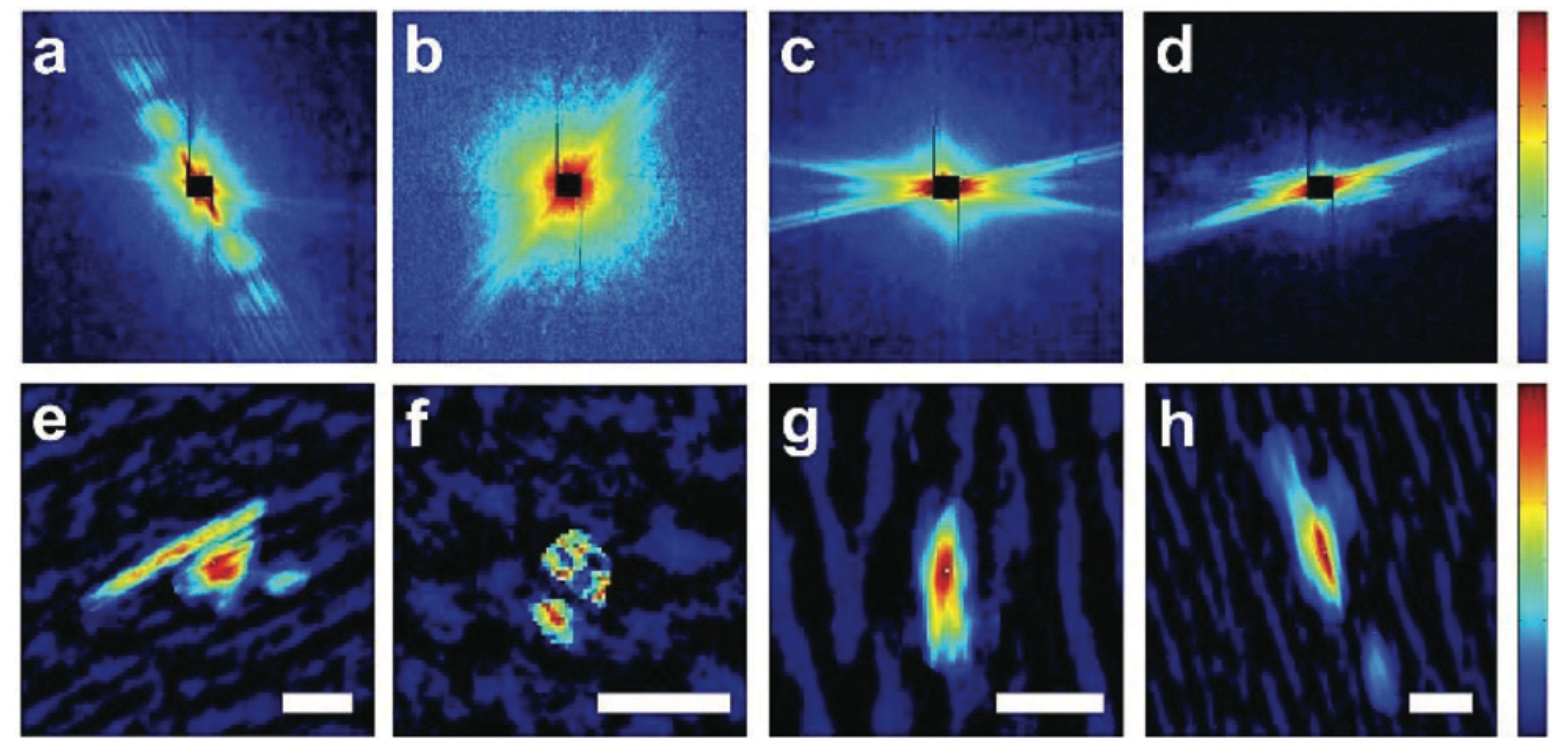

Fig. $3 \mathrm{CDI}$ patterns of four individual doxorubicin-containing lipo- somes. (a)-(d) Diffraction patterns; (e)-(h) their corresponding recon- structed images. Bars $=200 \mathrm{~nm}$.

Note that the diffraction patterns of Fig. 3 show only rodrelated features but no circular ones, as expected for the liposomes. To explain this point, we must again consider the role of $(\mathrm{NH} 4) 2 \mathrm{SO} 4$, from the precursor solution. Fig. 4 shows the results of simulation. Specifically, Fig. 4(c) shows a simulated diffraction pattern of a drug-containing liposome, modified from 
Fig. 4a by including a simulated circular diffraction pattern caused by the $\left(\mathrm{NH}_{4}\right)_{2} \mathrm{SO}_{4}(2 \mathrm{M}$ concentration) in the liposome. Fig. 4(d) shows the corresponding reconstruction, revealing the spherical liposome, which is not visible in the real reconstructions of Fig. 4(b). Fig. 4(e) and (f) show the simulated pattern and the reconstruction for a system similar to that of Fig. 4(c) and (d), but in a water solution with a sulphate concentration reduced to $500 \mathrm{mM}$. We no longer see features related to the liposomes.
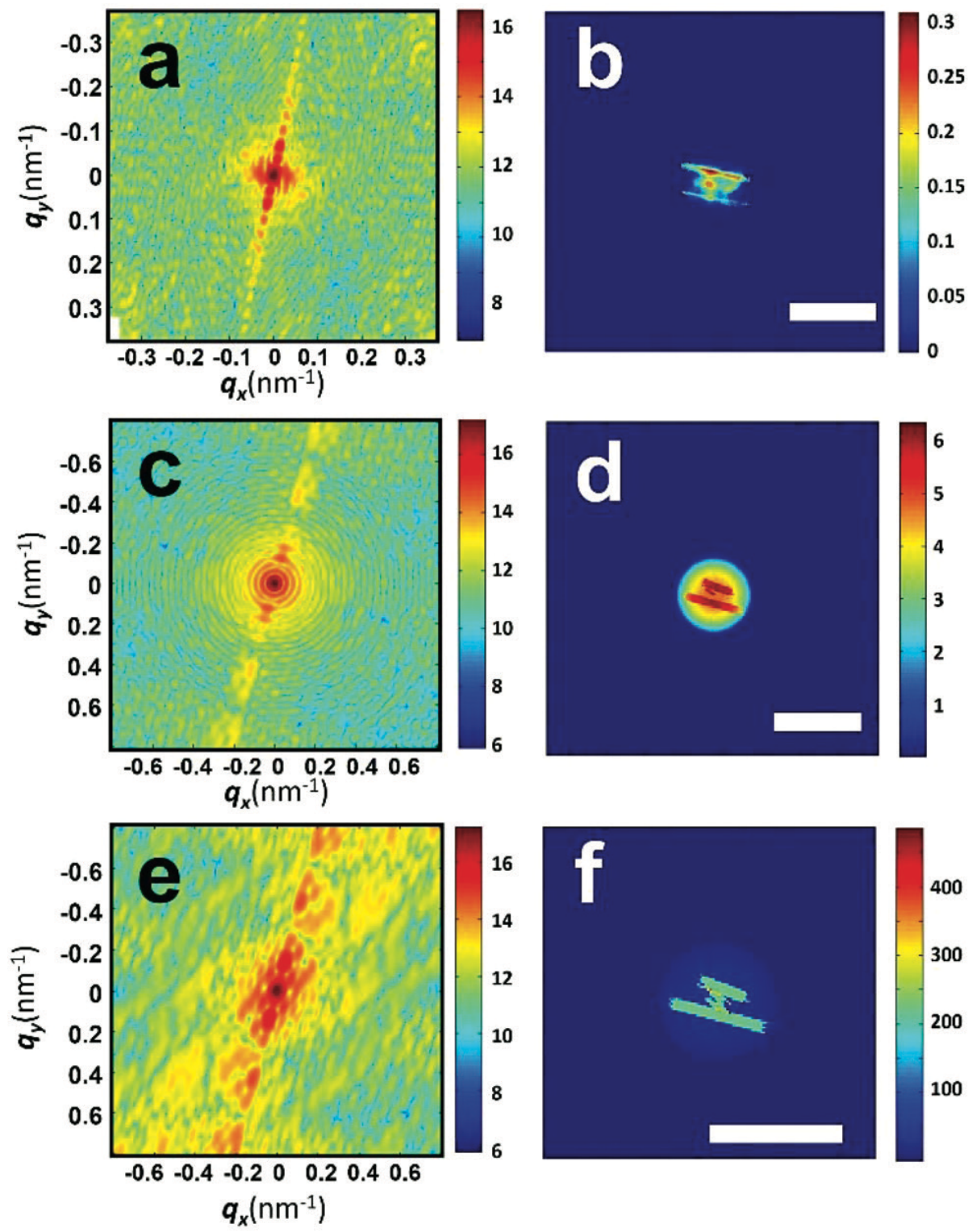

Fig. 4 Simulated CDI patterns of individual doxorubicin-containing liposomes and their corresponding reconstructed images. (a) and (b) are the diffraction pattern and the CDI reconstructed result experimentally obtained for a drug-containing liposome, respectively. (c) and (d) the results for a solution containing 2 M of $(\mathrm{NH} 4) 2 \mathrm{SO} 4$, as used in the pro- duction process. These simulations show that the liposome-related fea- tures should be visible, whereas in Fig. 4 they are not. (e) and (f) are the results for a (NH4)2SO4 solution concentration reduced to $500 \mathrm{mM}$ : the liposome-related features disappeared. Scale bars $=200 \mathrm{~nm}$.

For example, our data show that the concentration of $\left(\mathrm{NH}_{4}\right)_{2} \mathrm{SO}_{4}$ in blank liposomes is $\sim 2$ $\mathrm{M}$, whereas with drug rods the concentration reduced to $<1 \mathrm{M}$. Our measurements, including those for blank liposomes, therefore provide important chemical information of the solution, specifically on the ammonium sulphate concentration in the liquid within individual drugcontaining liposomes, difficult to obtain by other means. The measured reduction of ammonium sulphate concentration substantiates the incorporation of the sulphate into the 
rods during their formation and the subsequent depletion from the solution as previously hypothesized.

\section{Experimental}

We performed cryo-EM with a JEOL $2100 \mathrm{fx}$ instrument equipped with a direct detection device (DDD) $5 \mathrm{~K} \times 4 \mathrm{~K}$ pixel camera and a cryogenic sample holder. The specimens were prepared with a Leica EM GP instrument that rapidly froze the liposome particles in vitreous ice.

CDI tests were performed at the SACLA X-FEL using $3.9613 \mathrm{keV}$ photon pulses with 425.4 $\mu \mathrm{J}$ per pulse on the average, $10 \mathrm{fs}$ duration and a $10 \mathrm{~Hz}$ repetition rate. The pulses were focused on the sample, down to $1.3 \times 1 \mu^{2}$, using a Kirkpatrick-Baez (KB) mirror system.

The particle solution was placed in a micro-liquid enclosure array (MLEA) sample chip ${ }^{4}$ with $24 \times 24$ enclosures, sealed by $100 \mathrm{~nm}$ thick SiN membranes on the two sides. Each enclosure had a volume of $20 \times 20 \times 2 \mu \mathrm{m}^{3}$. The MLEA was placed in the vacuum system of the SACLA Multiple Application X-ray Imaging Chamber (MAXIC) instrument. ${ }^{33}$

The chip position was scanned with respect to the X-ray beam so that each enclosure was hit by only one X-FEL pulse per scan. If the enclosure contained a particle, this created a diffraction pattern before being destroyed.

The solution had a concentration corresponding to $\sim 1$ liposome per $1 \mu \mathrm{m}^{3}$. This minimized the probability of probing more than one liposome per pulse, which would otherwise adversely affect the CDI reconstruction. ${ }^{34,35}$ Note, however, that the concentration could not be too low, to avoid a large percentage of null diffraction results. We found that the above concentration was optimal, producing $>50 \%$ useable diffraction patterns corresponding to $>100$ successful reconstructions from each MLEA chip.

The diffraction patterns were recorded with a MPCCD (multiport charge-coupled device) octal sensor with a total area of $2399 \times 2399$ pixels (pixel size $=50 \times 50 \mu \mathrm{m}^{2}$ ). ${ }^{36}$ The sampledetector distance was $1.51 \mathrm{~m}$.

The CDI data analysis and reconstruction procedure were as follows. Firstly, since the diffraction pattern must be center-symmetric, we rotated the acquired pattern by 180 degrees and shifted the image pixel-by-pixel to find the origin by minimizing the L1 norm. After identifying the origin, we averaged the original pattern and the rotated one. Secondly, we cropped $471 \times 471$ and $601 \times 601$ pixels for the analyzed patterns. The corresponding estimated pixel resolutions of the reconstructed images were $20.0 \mathrm{~nm}$ and $15.7 \mathrm{~nm}$.

Thirdly, for each pattern the $\mathrm{GHIO}$ method $^{16}$ was applied to obtain a preliminary reconstruction. We then Fourier-transformed these reconstructions excluding the missing central speckle, and used the reverse transform to fill up the missing pixels. Finally, we repeated the first step but limited the image shifting to 0.1 pixels, obtaining a refined pattern. For each refined pattern, we performed a final reconstruction by combining GHIO and the Shrinkwrap algorithm (SW). A loose support was used to guarantee that no relevant signal was removed when applying the support constraint. For each iteration of the reconstruction, 16 initial random phases were generated to obtain 16 different reconstructed images. To generate a new support for the next iteration, the average of the 16 images was Gaussian- 
smoothed and a background was subtracted. Instead of finding a convergent support as in the conventional SW method, we forced the new support to be smaller (by <10 pixels) than the previous one.

We monitored the evolution of the 16 reconstructed images over 20 iterations. The support changed from loose to overcropped; the distribution of the 16 reconstructed images was accordingly modified from divergent to convergent, and then back to divergent. The final support was obtained when most reconstructed images were consistent. The GHIO reconstruction was then reiterated based on the final pattern and the final support. The reconstruction was terminated when the error metric stagnated.

\section{Conclusions}

The performances of the SACLA X-FEL were sufficient to extend the technique of individual nanoparticle imaging by CDI reconstruction from highly X-ray absorbing systems to weak absorbers. The results on blank liposomes were quantitatively consistent with those of cryoEM. Furthermore, X-FEL CDI detected doxorubicin nanorods enclosed in liposomes and measured their structural properties.

Our results are important in view of the optimization of the industrial drug loading in liposome nanoparticles as required for official certification for human use. In more general terms, they demonstrate that X-FEL CDI can now image individual nanoparticles with nominally low diffraction cross-sections, in a liquid environment and with $<100 \mathrm{~nm}$ size - and therefore it has a significantly broader scope than suggested by previous tests.

The present results are thus important in themselves, in particular since liposome nanocarriers are the most widely used ones at present and we can envision the future to other systems such as Au nanocarriers, even more so since the case of liposomes is particularly difficult with respect to other cases.

\section{Acknowledgements}

This research is supported by the National Science and Technology Program for Nanoscience and Nanotechnology, the Biomedical Nano-Imaging Core Facility at the NSRRC (National Synchrotron Radiation Research Center, Taiwan), Academia Sinica and the Ministry of Science and Technology (MOST 104-021001-09-02, MOST 105-0210-01-13-01, and MOST 106-0210-01-15-02). We thank Yoshitaka Bessho, Yasumasa Joti, Takashi Kimura and Yoshinori Nishino for their assistance in the experiments. Preliminary synchrotron measurements were performed at the NSRRC, Spring-8 (Japan) and the Pohang Light Source (PLS-II, Korea).

\section{References}

1. D. Pile, Nat. Photonics, 2011, 5, 456-457.

2. P. Emma, R. Akre, J. Arthur, R. Bionta, C. Bostedt, J. Bozek, A. Brachmann, P. Bucksbaum, R. Coffee and F.-J. Decker, Nat. Photonics, 2010, 4, 641-647.

3. T. Ishikawa, H. Aoyagi, T. Asaka, Y. Asano, N. Azumi, T. Bizen, H. Ego, K. Fukami, T. Fukui and Y. Furukawa, Nat. Photonics, 2012, 6, 540-544.

4. T. Kimura, Y. Joti, A. Shibuya, C. Song, S. Kim, K. Tono, M. Yabashi, M. Tamakoshi, T. Moriya and T. Oshima, Nat. Commun., 2014, 5, 3052.

5. M. Gallagher-Jones, Y. Bessho, S. Kim, J. Park, S. Kim, D. Nam, C. Kim, Y. Kim, O. Miyashita and F. Tama, Nat. Commun., 2014, 5, 3798. 
6. G. van der Schot, M. Svenda, F. R. Maia, M. Hantke, D. P. DePonte, M. M. Seibert, A. Aquila, J. Schulz, R. Kirian and M. Liang, Nat. Commun., 2015, 6, 5704.

7. T. Ekeberg, M. Svenda, C. Abergel, F. R. Maia, V. Seltzer, J.-M. Claverie, M. Hantke, O. Jönsson, C. Nettelblad and G. van der Schot, Phys. Rev. Lett., 2015, 114, 098102.

8. M. Watari, R. McKendry, M. Voegtli, G. Aeppli, Y. Soh, X. Shi, G. Xiong, X. Huang, R. Harder and I. K. Robinson, Nat. Mater., 2011, 10, 862-866.

9. D. Nam, J. Park, M. Gallagher-Jones, S. Kim, S. Kim, Y. Kohmura, H. Naitow, N. Kunishima, T. Yoshida, T. Ishikawa and C. Song, Phys. Rev. Lett., 2013, 110, 098103.

10. D. Papahadjopoulos, T. Allen, A. Gabizon, E. Mayhew, K. Matthay, S. Huang, K. Lee, M. Woodle, D. Lasic and C. Redemann, Proc. Natl. Acad. Sci. U. S. A., 1991, 88, 11460-11464.

11. A. Gabizon, R. Catane, B. Uziely, B. Kaufman, T. Safra, R. Cohen, F. Martin, A. Huang and Y. Barenholz, Cancer Res., 1994, 54, 987-992.

12. D. W. Northfelt, F. J. Martin, P. Working, P. A. Volberding, J. Russell, M. Newman, M. A. Amantea and L. D. Kaplan, J. Clin. Pharmacol., 1996, 36, 55-63.

13. J. R. Fienup, Appl. Opt., 1982, 21, 2758-2769.

14. V. Elser, J. Opt. Soc. Am., 2003, 20, 40-55.

15. H. H. Bauschke, P. L. Combettes and D. R. Luke, J. Opt. Soc. Am., 2002, 19, 1334-1345.

16. C. Chen, J. Miao, C. Wang and T. Lee, Phys. Rev. B: Condens. Matter Mater. Phys., 2007, 76, 064113.

17. T. M. Allen and P. R. Cullis, Science, 2004, 303, 1818-1822.

18. A. Samad, Y. Sultana and M. Aqil, Curr. Drug Delivery, 2007, 4, 297-305.

19. B. C. Keller, Trends Food Sci. Technol., 2001, 12, 25-31.

20. F. Arcamone, G. Cassinelli, G. Fantini, A. Grein, P. Orezzi, C. Pol and C. Spalla, Biotechnol. Bioeng., 1969, 11, 1101-1110.

21. R. I. Pakunlu, Y. Wang, M. Saad, J. J. Khandare, V. Starovoytov and T. Minko, J. Controlled Release, 2006, 114, 153-162.

22. T. Kubo, T. Sugita, S. Shimose, Y. Nitta, Y. Ikuta and T. Murakami, Int. J. Oncol., 2000, 17, 309-324.

23. A. A. Gabizon, Cancer Invest., 2001, 19, 424-436.

24. B. Uziely, S. Jeffers, R. Isacson, K. Kutsch, D. Wei-Tsao, Z. Yehoshua, E. Libson, F. M. Muggia and A. Gabizon, J. Clin. Oncol., 1995, 13, 1777-1785.

25. S. A. Abraham, D. N. Waterhouse, L. D. Mayer, P. R. Cullis, T. D. Madden and M. B. Bally, Methods Enzymol., 2005, 391, 71-97.

26. S. K. Hobbs, W. L. Monsky, F. Yuan, W. G. Roberts, L. Griffith, V. P. Torchilin and R. K. Jain, Proc. Natl. Acad. Sci. U. S. A., 1998, 95, 4607-4612.

27. Y.-M. Wu, C.-H. Wang, J.-w. Chang, Y.-y. Chen, N. Miyazaki, K. Murata, K. Nagayama and W.-H. Chang, J. Phys. D: Appl. Phys., 2013, 46, 494008.

28. Y.-M. Wu, J.-W. Chang, C.-H. Wang, K. Nagayama, N. Miyazaki, K. Murata and W.-H. Chang, Microsc. Microanal., 2015, 21, 2145-2146.

29. M. Almgren, K. Edwards and G. Karlsson, Colloids Surf., A, 2000, 174, 3-21.

30. I. V. Zhigaltsev, N. Maurer, Q.-F. Akhong, R. Leone, E. Leng, J. Wang, S. C. Semple and P. R. Cullis, J. Controlled Release, 2005, 104, 103-111.

31. N. Weiner, F. Martin and M. Riaz, Drug Dev. Ind. Pharm., 1989, 15, 1523-1554.

32. M. Kotlarchyk and S. H. Chen, J. Chem. Phys., 1983, 79, 2461-2469.

33. C. Song, K. Tono, J. Park, T. Ebisu, S. Kim, H. Shimada, S. Kim, M. Gallagher-Jones, D. Nam, T. Sato, T. Togashi, K. Ogawa, Y. Joti, T. Kameshima, S. Ono, T. Hatsui, S. Iwata, M. Yabashi and T. Ishikawa, J. Appl. Crystallogr., 2014, 47, 188-197.

34. Y. Takahashi, A. Suzuki, N. Zettsu, T. Oroguchi, Y. Takayama, Y. Sekiguchi, A. Kobayashi, M. Yamamoto and M. Nakasako, Nano Lett., 2013, 13, 6028-6032.

35. M. J. Bogan, D. Starodub, C. Y. Hampton and R. G. Sierra, J. Phys. B: At., Mol. Opt. Phys., 2010, 43, 194013.

36. M. Yabashi and T. Ishikawa, in XFEL/SPring-8 Beamline Technical Design Report ver. 2.0 Experimental facility group, SPring-8 joint project for XFEL, Hyogo, 2010, pp. 42-46. 